\title{
Le luxe et Internet : Evolutions d'un paradoxe
}

\author{
Angy Geerts* \\ Chargée de cours \\ Université de Mons - Belgique \\ Nathalie Veg-Sala** \\ Maître de Conférences \\ Université Paris Ouest Nanterre La Défense - France
}

* Université de Mons - Faculté Warocqué Economie et Gestion - Place warocqué 17, 7000 Mons - Belgique

Angy.geerts@umons.ac.be

** Université -Paris Ouest Nanterre La Défense - 200 avenue de la République, 92001 Nanterre Cedex

Nathalie.Veg-Sala@u-paris10.fr 


\title{
Le luxe et Internet : Evolutions d'un paradoxe
}

\section{Résumé :}

Alors que le luxe se caractérise par une image de rareté et de sélectivité, Internet repose sur le principe de diffusion et d'accessibilité. Face à cette opposition, les marques de luxe ont longtemps été sceptiques quant à la création d'un site qui leur serait propre et dans lequel il pourrait informer, communiquer voire vendre. Ce scepticisme se renforçait par les études menées dans les années 1990 et au début des années 2000 sur les perceptions des consommateurs et des managers. Mais 20 ans après, face aux évolutions socioculturelles et technologiques, très peu de travaux ont été menés sur le sujet; la dernière recherche de référence datant de 2003. L'objectif de cet article est d'analyser l'évolution des perceptions des managers et des consommateurs face à l'utilisation d'Internet dans le luxe. Sur la base d'une revue des travaux fondateurs sur ces thématiques et d'une triple méthodologie, qualitative, quantitative et d'analyse de contenu, cette recherche discute des principales évolutions de la relation entre le monde du luxe et celui d'Internet et ce, à trois niveaux : les perceptions des consommateurs, les perceptions des managers et le contenu des sites des marques de luxe. Une réelle accélération de l'acceptation d'Internet est mise en évidence et le paradoxe entre luxe et Internet tend à se déplacer progressivement vers les questions de la mise en place de l'e-commerce.

\section{Mots Clés :}

Internet, e-commerce, luxe, consommateurs, managers

\section{Luxury and Internet: Evolutions of a paradox}

\begin{abstract}
:
While luxury is characterized by an image of rarity and selectivity, Internet relies on a concept of accessibility. Regarding this paradox, luxury brands have been reluctant for a long time as far as the implementation of their own website is concerned. This scepticism was strengthened by the studies led in the 1990s and the beginning of 2000s, regarding the consumers and managers' perceptions. But 20 years later, while the sociocultural and technological evolutions were numerous, very few academic research have been conducted on that topic; the last important research has been led in 2003. The purpose of this paper is to analyze the evolution of the managers and the consumers' perceptions regarding the use of Internet in the luxury sector. On the basis of a literature review of the founding papers on these themes and of a triple methodology, qualitative, quantitative and content analysis, this research discusses the main evolutions of the relation between the luxury world and the Internet. The evolutions have been analyzed at three levels: the consumers' perceptions, the managers' perceptions and the contents analysis of luxury brands websites. A real acceleration of the acceptance of Internet is emphasized and the paradox between luxury and Internet is moving gradually towards the questions of the implementation of the e-commerce.
\end{abstract}

\section{Key words:}

Internet, E-shopping, luxury, consumers, managers 
Le secteur du luxe est en constante évolution. Il se déploie, innove et recherche de nouveaux leviers de croissance. Internet, de par son ampleur et son ancrage dans le quotidien des individus (Lemoine, 2012), constitue une opportunité de développement et de dynamisme. Pourtant lorsqu'Internet apparaît au grand public en 1994, les marques de luxe sont frileuses face à son utilisation. Elles redoutent une perte de contrôle, une dilution de leurs valeurs d'exclusivité et de rareté. Cette hésitation est renforcée par les recherches des années 1990 qui avaient essentiellement pointé du doigt les risques de l'utilisation d'Internet dans le luxe (Nyeck et Houde, 1996 ; Nyeck et Roux, 1997).

Vingt ans après, les évolutions ont été rapides et nombreuses sur ce marché. Toutes les marques de luxe possèdent leur propre site Internet. Et au-delà des sites traditionnels, certaines maisons de luxe se lancent dans l'e-commerce. Selon le cabinet Percepta (groupe Xerfi, 2010), les ventes en ligne représentent aujourd'hui près de $5 \%$ des ventes totales dans le luxe contre $3,8 \%$ en 2010 et $0,8 \%$ en 2005 . Les prévisions pour 2015 parlent d'un doublement du marché du luxe en ligne pour représenter $10 \%$ des ventes.

Par ailleurs, alors que le marché subit de nombreuses transformations, les recherches ont stagné depuis quelques temps. La plupart remonte au milieu des années 1990. Le dernier article académique de référence sur ce thème date de 2003 (Dall'Olmo Riley et Lacroix, 2003). Dans cette recherche, les auteurs font le point simultanément sur les attitudes des consommateurs et des managers mais font également un état des lieux des contenus des sites Internet des marques de luxe. Mais en dix ans, les attitudes des managers et des consommateurs ont-elles changé ? Quelles évolutions peut-on également observer sur les sites Internet des marques de luxe ?

Réactualiser ces données et développer une meilleure compréhension des internautes (Lemoine, 2012) tout comme celle des experts du secteur apparaissent essentielles afin de mieux renseigner les marques sur les stratégies à adopter. C'est pourquoi cet article a pour objectif d'analyser comment Internet et le e-commerce s'insèrent aujourd'hui dans le secteur du luxe.

Afin de répondre à ce questionnement, cet article s'articule autour des spécificités des marques de luxe et sur les résultats des travaux qui se sont intéressés à Internet et au ecommerce dans le secteur du luxe. Dans l'objectif d'étudier les évolutions et faire des comparaisons notamment avec la recherche de Dall'Olmo Riley et Lacroix (2003), la méthodologie développée s'appuie sur une triple étude : (1) une analyse qualitative sur les perceptions des managers, (2) une double analyse, qualitative et quantitative, sur les perceptions des consommateurs et (3) une analyse de contenu des sites Internet des marques de luxe. Les résultats sont ensuite discutés afin de donner de nouvelles perspectives aux managers des marques de luxe.

\section{Les marques de luxe : entre sélectivité et diffusion}

Le luxe peut être défini comme une façon d'être, une manière de vivre mais aussi une façon d'acheter, de consommer et d'utiliser (Bechtold, 1991 ; Roux et Floch, 1996 ; Castarède, 2004 ; Nyeck, 2004). Le luxe est pluriel et aborde plusieurs facettes allant du besoin de paraître aux yeux de la société (luxe traditionnel) à la recherche de sensations et de valeurs expérientielles personnelles (luxe émotionnel) (Lipovetsky et Roux, 2003). Dans le luxe, la part d'immatériel est immense et l'univers créé est primordial (Bechtold, 1991). Dans ce contexte, les marques jouent un rôle fondamental. Ce sont elles qui véhiculent les récits et racontent des histoires (Nyeck, 2004). Elles doivent être le vecteur des valeurs d'unicité, de rareté et de sélectivité. A travers leurs racines culturelles et géographiques mais également leur fonction ontologique, 
les marques de luxe doivent permettre de recréer l'écart et maintenir leur image inaccessible (Keller, 2009). Tous les aspects du marketing mis en place pour les marques de luxe doivent assurer une expérience d'achat et de consommation unique (Keller, 2009).

Malgré un fort besoin de contrôle, les marques appartenant au secteur du luxe doivent, tout autant que les autres, essayer sans cesse de se développer. La recherche de nouveaux leviers de croissance est essentielle. Les marques de luxe font face à de nombreux paradoxes, entre sélectivité et diffusion, rareté et expansion (Bechtold, 1991 ; Roux, 1994 ; Nueno et Quelch, 1998 ; Heilbrunn, 2002), tant au niveau de leur communication que de leur distribution. Les marques de luxe doivent mettre en avant une image élitiste avec notamment un choix de communication sélective (Allérès, 1991, 1997 ; Chevalier et Mazzolovo, 2008), protégeant leur sélectivité et leur permettant de rester discrètes. Mais la communication des marques de luxe est également essentielle afin d'accroître leur notoriété, montrer qu'elles existent, qu'elles sont présentes et fortes. Il en va de même avec la distribution des produits de luxe, spécifique à bien des égards (Kapferer et Bastien, 2008). Elle est fondée à la fois sur le prestige et la rareté des produits vendus. L'expérience vécue en boutique est essentielle (Atwal et Williams, 2009), tout comme l'émotion et le sensoriel. Mais la distribution doit également couvrir de manière rentable la majeure partie de la cible, dans son pays et à l'étranger. La gestion de ces paradoxes se renforce dès lors que les marques de luxe développent une réflexion sur l'intégration d'Internet comme outil de communication et/ou de distribution.

\section{Les marques de luxe et Internet : un début contrasté}

A l'exemple de Chanel ou de Hermès, certaines marques de luxe ont longtemps hésité à utiliser Internet, affichant de lourdes réserves à son égard (Larbanet et Ligier, 2010). Cette incertitude est liée aux risques associés à cet outil de communication en sus des avantages certains mis en avant dans les recherches des années 1990 et du début des années 2000.

\subsection{Des avantages incontestés}

$\mathrm{Du}$ point de vue des managers, Internet constitue un outil économique en termes de communication car les coûts sont moins élevés que pour les autres canaux. De plus, les managers pensent qu'Internet est favorable car il permet de fournir un maximum d'informations simultanément à de nombreuses personnes. Internet offre la possibilité de diffuser des messages accessibles par tous (Chaffey et al, 2003 ; Okonkwo, 2009, 2010). Internet est un support quasi «illimité » où il est possible de montrer aux consommateurs ce qu'est la marque, à travers des photos, des vidéos, des présentations de produits (Chaffey et al, 2003 ; Okonkwo, 2009, 2010).

Pour les consommateurs, Internet est perçu comme un outil dynamique et novateur (Nyeck et Roux, 1997). Les consommateurs peuvent facilement avoir des renseignements sur la marque à n'importe quelle heure du jour et de la nuit, sans même se déplacer (Nyeck et Roux, 1997). Pour 22\% d'entre eux, ils recherchent des informations sur la marque, pour $21 \%$, des informations sur le produit, et $12 \%$ des consommateurs souhaitent trouver un conseil personnalisé (Dall'Olmo Riley et Lacroix, 2003). Notons toutefois qu'à cette époque, dans $40 \%$ des cas, les répondants n'ont pas été en mesure de trouver ce qu'ils recherchaient. De plus, Internet est perçu par les consommateurs comme un outil complémentaire aux autres médias. Par rapport à une brochure classique, Internet permet de construire une relation plus personnelle et plus intime entre la marque et le consommateur lorsque celui-ci est impliqué et 
Geerts, Angy et Nathalie Veg-Sala (2014), "Le luxe et Internet : Evolutions d'un paradoxe »,

Management \& Avenir (accepté le 25 février 2014)

intéressé par la marque (Nyeck et Roux, 1997). Enfin, Internet ne semble pas modifier l'image que les consommateurs ont du luxe (Nyeck et Roux, 1997). Ils apprécient particulièrement les sites des marques de luxe avec une esthétique irréprochable et qui communiquent de façon claire (Dall'Olmo Riley et Lacroix, 2003).

\subsection{Des limites relatives}

En complément des avantages identifiés, Internet est vu par les managers comme un outil de communication risqué car les consommateurs peuvent en prendre le contrôle (Okonkwo, 2009). De par la création de forums ou bien de blogs, les Internautes ne sont plus uniquement passifs face à la communication des marques mais peuvent en être les auteurs. Les consommateurs, quant à eux, perçoivent Internet comme une interface qui peut être impersonnelle et complexe (Nyeck et Roux, 1997). Ils peuvent regretter le manque de considération face à un outil où tout le monde semble être traité de la même façon (Okonkwo, 2009). Ces impressions plutôt négatives sont à relativiser en fonction des catégories de produits des marques (Nyeck et Roux, 1997). Internet ne serait pas adapté pour véhiculer l'image des produits dont la teneur en rêve est la raison d'être (comme pour les cosmétiques et les parfums). Les montres et accessoires ne souffrent pas du support Internet même si le catalogue reste plus prestigieux.

Les perceptions des consommateurs dépendent également de leur âge (Nyeck et Roux, 1997). Il existe un clivage entre la perception des plus de 45 ans, jugeant que le luxe est incompatible avec Internet, et les plus jeunes, enclins à s'adapter à ce média de communication.

\section{Les marques de luxe et le e-commerce : une présence contestée}

Au-delà de l'analyse d'Internet comme support de communication, certaines recherches ont étudié un peu plus récemment les perceptions des managers et des consommateurs face au ecommerce dans le secteur du luxe. Durant cette période, l'e-commerce représentait à la fois une opportunité et une menace (Dall'Olmo Riley et Lacroix, 2003).

\subsection{Des opportunités identifiées}

Alors que les marques de luxe développent généralement des réseaux sélectifs de boutiques physiques, Internet offre la possibilité d'acheter, à des consommateurs qui n'ont pas d'accès proche à un des rares magasins de luxe (Seringhaus, 2005 ; Larbanet et Ligier, 2010). Ces boutiques virtuelles peuvent également convenir aux consommateurs qui se sentent intimidés par le fait de pénétrer dans les magasins des marques de luxe (Seringhaus, 2005 ; Larbanet et Ligier, 2010). Internet permet d'offrir de nombreux services aux consommateurs tels que la personnalisation des produits, le paiement en ligne et la livraison (Larbanet et Ligier, 2010). Un site d'e-commerce fournit des informations d'une façon nouvelle, les photos et les vidéos aidant à compenser le manque de contact (de Chernatony, 2001). Les marques de luxe doivent cependant faire attention à maintenir leur essence et rester cohérentes avec leur identité (de Chernatony, 2001 ; Veg-Sala et Geerts, 2011). Grâce à ces nombreux avantages, la demande pour la vente du luxe en ligne au niveau mondial est en pleine croissance (Okonkwo, 2010). Cependant, seul 5\% des visiteurs vont sur les sites des marques de luxe dans le but d'y réaliser un achat (Dall'Olmo Riley et Lacroix, 2003).

\subsection{Des risques potentiels majeurs}


Internet étant un canal de distribution à grande ampleur, les marques de luxe qui s'y exposent peuvent être face à un risque accru de diffusion de contrefaçons (Kapferer et Bastien, 2008). Les marques redoutent aussi une potentielle cannibalisation du e-commerce par rapport à leurs magasins traditionnels (Larbanet et Ligier, 2010 ; Kapferer et Bastien, 2008). Au delà, cette façon de distribuer oblige les marques de luxe à révéler leurs prix, donnant la possibilité aux consommateurs de faire plus facilement des comparaisons avec les concurrents (Larbanet et Ligier, 2010) ; or le luxe est superlatif et non comparatif (Kapferer et Bastien, 2008). Par ailleurs, les managers des marques de luxe craignent la perte de spontanéité de l'achat sur Internet en raison de la prise de décision consciente (Dall'Olmo Riley et Lacroix, 2003).

Notons que les opinions des managers sont contrastées. Trois groupes de managers sont identifiés dans la typologie développée par Nyeck et Roux en 1997 : les conservateurs, les sceptiques et les ouverts. Les conservateurs considèrent comme risqué l'utilisation d'Internet et du e-commerce dans le luxe et défendent une position claire sur l'importance de la communication unidirectionnelle et la distribution sélective. Les sceptiques pensent qu'Internet constitue un élément d'avenir mais leurs craintes les poussent à poursuivre leur politique actuelle d'élitisme. Enfin, les ouverts considèrent Internet comme une réelle opportunité, permettant de donner un dynamisme et une image novatrice des marques de luxe. Ils pensent que l'intégration d'un e-commerce est possible dans le luxe, permettant à certains clients de faciliter leurs achats et de rajouter du confort dans leur processus de consommation. La diversité d'opinion des managers se traduit dans le contenu des sites. L'analyse menée par Dall'Olmo Riley et Lacroix (2003) s'est penchée sur les caractéristiques générales des sites et leur aspect visuel. Une forte hétérogénéité des sites Internet est observée. A cette époque, la plupart étaient des sites esthétiques et soignés même si seulement $70 \%$ d'entre eux étaient opérationnels. Il en ressort que seuls $15 \%$ des sites proposaient la vente en ligne et $11 \%$ proposent des zones réservées aux membres privilégiés. Par contre 60\% des sites analysés offraient une possibilité de contact avec la marque (par exemple par e-mail).

Le manque d'expérience apparaît comme une limite fondamentale de l'utilisation de l'ecommerce pour les marques de luxe (Allérès, 1991, 1997 Chevalier et Mazzalovo, 2008 ; Okonkwo, 2009, 2010). La vente en ligne ne peut pas pour les consommateurs véhiculer les émotions et le sensoriel pourtant essentiels dans le luxe (Nyeck et Houde, 1996 ; Kapferer et Bastien, 2008). Les consommateurs ont de ce fait une préférence pour l'expérience en magasin (Dall'Olmo Riley et Lacroix, 2003). Le risque d'insécurité du paiement en ligne perçue par certains consommateurs renforce la méfiance des marques face à l'adoption du ecommerce (Dall'Olmo Riley et Lacroix, 2003). Ces divers risques sont à relativiser en fonction des catégories de produits (Nyeck et Roux, 1997 ; Dall'Olmo Riley et Lacroix, 2003 ; Seringhaus, 2005). L'hôtellerie de luxe se vendrait très bien sur le net, comme les accessoires de mode, les vins ou spiritueux. Par contre, l'e-commerce ne conviendrait pas pour la haute joaillerie.. Selon l'âge, les attitudes des consommateurs face au e-commerce dans le luxe varie également (Nyeck et Roux, 1997); les plus de 45 ans étant les plus sceptiques à la vente en ligne.

\section{Méthodologie de recherche}

\subsection{Objectifs et contexte de la méthodologie de recherche}


L'objectif de la méthodologie est double. Il s'agit: (1) de faire un état des lieux actuel et complet sur les liens entre marques de luxe et Internet puis (2) d'analyser les évolutions par rapport aux travaux antérieurs remontant à une dizaine, voire une vingtaine d'années. Dans ce but, la méthodologie utilisée suit celles des recherches fondatrices sur ces thématiques, à savoir celles de Nyeck et Houde (1996), Nyeck et Roux (1997) et de Dall'Olmo Riley et Lacroix (2003). Elle se décompose en trois parties :

- une étude qualitative auprès de managers du secteur du luxe ;

- une double étude, qualitative et quantitative, auprès de consommateurs de luxe et,

- une analyse de contenu des sites des marques de luxe.

\subsection{L'étude qualitative sur les managers}

Afin de saisir la vision actuelle des professionnels du secteur du luxe concernant l'utilisation d'Internet et du e-commerce, huit entretiens semi-directifs ont été réalisés auprès de managers de plusieurs maisons de luxe (Chanel, Hermès et Van Cleef \& Arpels) et d'entreprises du monde digital travaillant pour les marques de luxe (Emakina, SCS, Genius, Account Ibis Advertising). Les principaux thèmes abordés lors de ces entretiens sont : les perceptions concernant le lien entre luxe, Internet et e-commerce, les actions menées dans les entreprises, leurs futures perspectives et les caractéristiques qui leur paraissaient indispensables aujourd'hui pour le luxe sur Internet. Les entretiens ont été réalisés en face à face, dans les bureaux des interviewés, par téléphone ou bien par échanges électroniques, entre le début de l'année 2011 et le milieu de l'année 2012. Ils ont duré entre 30 min et 1h30. Intégralement retranscrits, les entretiens ont fait l'objet d'une analyse de contenu manuelle, selon une analyse thématique catégorielle, consistant à calculer et à comparer les fréquences des éléments et thèmes évoqués et à les regrouper en catégories significatives.

\subsection{Les études qualitative et quantitative sur les consommateurs de luxe}

Les études qualitative et quantitative se sont effectuées en Belgique et ont porté sur les clients de l'enseigne Francis Ferent. Il s'agit d'une chaîne de boutiques de prestige multimarques. Elle réunit des marques de luxe de prêt-à-porter, de maroquinerie, de chaussures et d'accessoires: Dolce \& Gabbana, Miu Miu, Prada, Jimmy Choo, Stella Mc Cartney, Givenchy, Fendi, Burberry... Quatre des cinq boutiques se situent à Bruxelles et une est à Knokke. L'enquête a été réalisée juste avant la mise en place de la vente en ligne chez F. Ferent en 2010.

L'étude qualitative se base sur un focus groupe composé de quatre hommes et de quatre femmes âgés de 30 à 60 ans et tous consommateurs de luxe, clients de l'enseigne F. Ferent. La discussion a eu lieu dans un restaurant où ont été conviés les participants pour le petit déjeuner, afin d'assurer une ambiance conviviale. Le guide d'entretien aborde plusieurs thèmes : leur vision du luxe, la distribution du luxe, Internet et le e-commerce. Le focus groupe a été retranscrit et a fait l'objet d'une analyse de contenu thématique catégorielle.

L'étude quantitative consiste en la diffusion d'un questionnaire auprès de consommateurs de luxe. Le questionnaire reprend les items les plus cités par les consommateurs lors de la phase qualitative et se base sur les thèmes suivants : le luxe, la distribution, Internet et l'ecommerce. L'échantillon est composé de 195 personnes, clientes des boutiques F. Ferent, interrogées à l'intérieur ou à la sortie de ces magasins par des enquêtrices formées au préalable. L'échantillon est structuré comme suit : $76,4 \%$ de femmes et $23,6 \%$ d'hommes ; $25 \%$ ont moins de 25 ans ; $28 \%$ sont dans la tranche $25-34 ; 21,5 \%$ dans la tranche $35-44$; 
Geerts, Angy et Nathalie Veg-Sala (2014), "Le luxe et Internet : Evolutions d'un paradoxe ", Management \& Avenir (accepté le 25 février 2014)

$12,8 \%$ dans la tranche $45-54$ et $11,8 \%$ ont plus de 55 ans. $36 \%$ des interrogés sont des employés ; $15 \%$ des étudiants ; $12,3 \%$ des cadres ; $12,8 \%$ des indépendants et presque $10 \%$ sont sans emplois. La structure de l'échantillon s'apparente à celle de la clientèle des magasins F. Ferent. Les résultats ont été traités sous le logiciel SPSS.

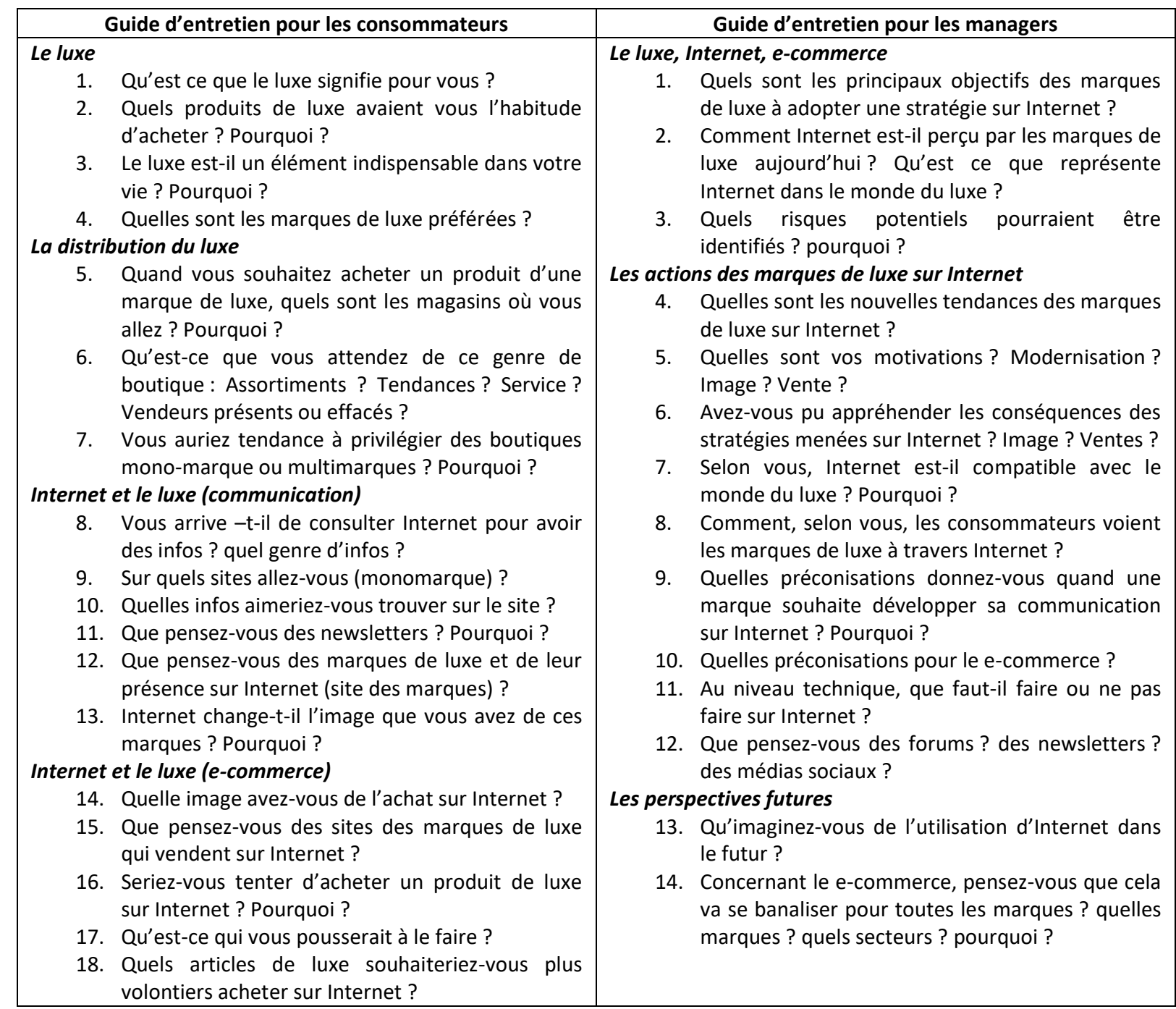

\subsection{L'analyse de contenu des sites des marques de luxe}

L'analyse de contenu a été effectuée sur 81 sites de marques de luxe dans le secteur du prêt-àporter, des accessoires et de la joaillerie/horlogerie. Le choix des marques s'appuie sur les critères suivants : les marques appartiennent soit au Comité Colbert, soit aux principaux groupes de luxe (LVMH, Kering, Richemont) ou bien sont citées dans les articles de recherche sur le luxe (Phau et Prendergast, 2000 ; Dubois et Laurent, 1993; Kapferer et Bastien, 2008 ; Lipovetsky et Roux, 2003 ; Okonkwo, 2007, 2010 ; Chadha Husband, 2006). Les sites Internet ont été audités sur la base d'une grille d'analyse validée par 6 experts du luxe et d'Internet. La grille abordait des sujets tels que la cohérence et l'esthétique de la marque, la politique produit, prix, distribution et communication sur le site, ainsi que l'interactivité et l'utilisabilité du site. Le codage de la grille est binaire attestant de la présence ou non de la variable sur le site audité. A partir de ce codage, une analyse factorielle en 
Geerts, Angy et Nathalie Veg-Sala (2014), "Le luxe et Internet : Evolutions d'un paradoxe ",

Management \& Avenir (accepté le 25 février 2014)

composantes principales (ACP) a pu être menée. En effet, Jolliffe (2002) indique qu'une analyse en composantes principales, a priori construite pour traiter des données continues, peut être utilisée sur des données qualitatives. Il faut noter que l'ACP d'un tableau formé de variables binaires donne les mêmes résultats que l'Analyse Factorielle des Correspondances Multiples (AFCM) (Meyer et al., 2010). Sur cette base, une analyse typologique a été effectuée, de type classification ascendante hiérarchique en utilisant le logiciel SPAD. L'algorithme utilisé par SPAD est celui de Ward. Cette méthode est l'une des plus précises quand un indicateur de distance euclidienne est utilisé. Cette analyse a donné lieu à une classification en quatre groupes, le choix du nombre de groupe est effectué sur base de l'indice d'homogénéité qui maximise la variance intergroupe par rapport à la variance totale. Les groupes ont été interprétés en fonction des caractéristiques des sujets qui les composent et des variables qui ont servi à les identifier (Jolibert et Jourdan, 2011).

\section{Résultats des études}

\subsection{La perspective des managers}

- Une présence essentielle et obligatoire pour communiquer les valeurs des marques

Pour les managers, Internet est aujourd'hui considéré comme un outil de communication incontournable. L'ensemble des experts interrogés y est favorable et ne se pose même plus de question sur la nécessité pour une marque de luxe d'avoir son propre site Internet: "Il est primordial pour les Maisons de luxe de développer leur univers et leur essence sur le net » (VCA). "Il est important que le site Internet soit cohérent avec les valeurs offline de la marque de luxe » (Genius).

- L'utilisation optimisée et maximisée des nouvelles technologies

Selon les managers interrogés, il est important que les sites Internet des marques de luxe soient développés selon les techniques les plus adéquates. Notamment, "cela pose un gros problème si un site d'une marque de luxe est aujourd'hui en Flash car il ne sera pas lisible sur l'iPad» (Chanel). Un besoin d'adaptation des moyens de connexion est recommandé : "Je suggérerais d'insérer la possibilité de choisir entre: "allez sur le site HTML ou FLASH" » (Emakina). Les éléments techniques liés au son ou à l'image sont décisifs : "Pour que le consommateur soit immergé dans l'histoire de la marque, elle utilise de plus en plus les vidéos»(Genius). En ce qui concerne la musique comme élément technique, il semble qu'elle soit « peu à peu abandonnée sur le net. Les internautes sont peu friands des sites qui lancent automatiquement une bande son car si l'internaute est sur plusieurs sites en même temps un risque de bug est à craindre » (Genius). L'actualisation des sites (tant sur leur contenu que sur leur utilisabilité) est essentielle : «les mises à jour du site doivent être très régulières » (VCA).

- Une attention portée sur les relations «marque / consommateur »

Les managers sont intéressés par les liens qu'il est possible ou non de tisser sur Internet entre les marques de luxe et les consommateurs : "L'interaction avec la marque est notamment essentielle pour l'actualité »(SCS). Les réflexions portent sur les outils à mettre en place. Concernant l'insertion de forum, les avis sont relativement négatifs. Les managers sont plutôt sceptiques énonçant que : "s'il y a un forum, cela ne correspond pas à un positionnement pertinent pour un site de luxe» (Emakina) ou bien que «les forums sont peu utilisés aujourd'hui au détriment des médias sociaux»(Genius). L'utilisation de forums semble ne 
Geerts, Angy et Nathalie Veg-Sala (2014), "Le luxe et Internet : Evolutions d'un paradoxe ", Management \& Avenir (accepté le 25 février 2014)

pas correspondre au positionnement des maisons de luxe : "J'ai du mal à imaginer des clientes du luxe partager leur expérience sur un forum... je trouve cela assez cheap » (Chanel). Des avis défavorables sont également cités pour l'utilisation de newsletters : «où il faut faire absolument attention à la fréquence d'envoi » (Chanel). Concernant l'utilisation des médias sociaux, des précautions sont à prendre pour les marques de luxe : "Cela doit être effectué dans la discrétion car il faut faire attention à la population que l'on vise »(SCS). Certains managers notent que pour favoriser les relations entre marques de luxe et consommateurs, tout en restant cohérent avec les valeurs du luxe, d'autres moyens sont à privilégier, comme la création de clubs privés, où seuls les clients peuvent y accéder : «un club parait plus adapté à un site de luxe qu'un programme de fidélisation » (Emakina).

- L'e-commerce pour les marques de luxe

L'utilisation du e-commerce dans le secteur du luxe reste encore une question très ambiguë, illustrée dans l'étude par les avis beaucoup moins explicites et moins nombreux. Les managers constatent que la vente en ligne des marques de luxe est de plus en plus développée : "l'e-shopping de luxe rentre de plus en plus dans les mours » (SCS) et semble être un acquis pour le futur, "comme une tendance qui va se confirmer dans le temps" (Emakina). Les managers réfléchissent aux stratégies connexes au e-commerce dans le luxe : «faut-il avoir la présence d'exclusivités online? peut-on "réserver» des produits ou bien prendre rendez-vous? »(VCA). Les questions portent sur la façon de mettre en place le ecommerce : "pour moi il n'est pas nécessaire d'avoir un site e-commerce intégré au site image de la marque, mais les liens doivent être clairs et faciles afin que le basculement entre les deux sites soit rapide »(SCS). La question des programmes de fidélité récolte des avis défavorables : «ça ne me semble pas très luxe comme positionnement » (Emakina); même si cela permet de mettre en place des «politiques de CRM plus efficaces»(SCS). Enfin, certaines idées émergent pour les marques qui ne souhaiteraient pas encore développer d'ecommerce. Il s'agit des «wishlists»: «cette possibilité permet aux consommateurs de s'impliquer davantage dans l'univers de la marque et d'augmenter son envie d'aller acheter des produits de la marque»(SCS). Cela a des avantages pour la marque: "grâce aux wishlists, les marques ont accès à des informations pertinentes et personnalisées...» (Chanel).

\subsection{La perspective des consommateurs}

- Résultats de l'étude qualitative

Les consommateurs interrogés semblent avoir largement intégré Internet comme vecteur de communication pour les marques de luxe. La première utilisation d'Internet pour les répondants est la recherche d'informations sur les nouveautés : "J'aime aller sur les sites pour voir si la nouvelle collection est arrivée » (Claudine, 49 ans) ; "Quand j'ai envie de me faire plaisir, je surfe sur les différents sites pour voir quelle marque pourrait faire mon bonheur» (Ania, 35 ans). Certains s'abonnent aux newsletters afin d'être certains d'obtenir les dernières actualités des marques. Leur seule crainte est d'être submergé d'e-mails de la marque : "J'ai quand même toujours peur de me faire bombarder de pubs et d'e-mails quand je m'inscris » (Afrim, 30 ans).

Concernant le e-commerce, les répondants montrent une large réticence à l'achat en ligne. Différents motifs ont été invoqués: le besoin de voir, de toucher ou d'essayer le produit ; la perte de la notion de plaisir associée à l'achat: "J'associe Internet au travail, quand je fais $d u$ shopping c'est pour mon loisir, en achetant en ligne j'aurais l'impression de perdre un 
peu du plaisir que j'ai à l'achat» (Claudine, 49 ans). Le manque de confiance dans l'achat en ligne reste un élément important pour quelques consommateurs : "Malgré les progrès en la matière, je ne suis toujours pas rassurée en ce qui concerne l'achat en ligne, et le fait d'encoder le numéro de ma carte de crédit » (Donatienne, 47 ans) ; " Pour ma part, j'ai peur de ne pas être satisfait de la qualité ou de recevoir une contrefaçon, surtout si j'achète un produit de luxe! »(Afrim, 30 ans).

- Résultats de l'étude quantitative

D'après l'étude menée, $63,6 \%$ des consommateurs interrogés recherchent des informations sur le luxe. $16,7 \%$ des consommateurs s'intéressent aux nouvelles collections, $14 \%$ à la visualisation des produits et à leurs spécificités, 10,2\% au prix, 13,2\% aux points de vente, $12,8 \%$ cherchent les nouvelles tendances. Seuls $33,1 \%$ d'entre eux sont abonnés à des newsletters de marques de luxe.

Concernant l'image des marques de luxe, $63,4 \%$ des consommateurs de luxe sont tout à fait d'accord sur l'importance de la présence des marques de luxe sur Internet. Toujours dans cette perspective, seuls $11,9 \%$ des consommateurs pensent qu'Internet est inadapté aux codes de l'univers du luxe et qu'il dégrade ces valeurs. Les analyses croisées montrent que les attitudes face à l'utilisation d'Internet par les marques de luxe ne dépendent pas de l'âge des individus $\left(X^{2}=2,275 ; p=0,321\right)$.

Parmi les consommateurs interrogés, 20,3\% achètent des produits de luxe sur Internet. Les achats en ligne de produits de luxe concernent principalement: la maroquinerie (32\%), le prêt-à-porter $(28 \%)$, les chaussures $(26 \%)$ et les parfums $(10 \%)$. L'achat de joaillerie et de vins ne représente au total que $4 \%$ des achats en ligne. Les principales raisons mises en avant par les consommateurs de luxe pour expliquer qu'ils n'achètent pas leurs produits sur Internet sont : leur préférence pour aller dans les magasins $(81,4 \%)$, le besoin de toucher et voir le produit $(83 \%)$, le manque de confiance lié aux achats en ligne $(41,2 \%)$. Enfin, les attitudes face à l'achat en ligne de produits de luxe ne dépendent pas de l'âge des individus $\left(X^{2}=0,121\right.$ $; p=0,728)$. Ces éléments sont en concordance avec les résultats du focus groupe.

\subsection{Les sites des marques de luxe : proposition d'une typologie}

L'analyse factorielle en composantes principales effectuée sur le contenu des sites audités met en avant trois axes. Le premier axe (représentant $26,11 \%$ de la variance expliquée) définit la présence d'E-shopping; le second axe (représentant 9,93\% de la variance expliquée) définit l'interactivité des sites et enfin le troisième axe (représentant 6,72\% de la variance expliquée) définit l'utilisabilité des sites Internet. Sur la base de ces axes, une typologie de sites de marques de luxe a été identifiée. Quatre groupes ont été mis en évidence.

La première classe, appelée E-shop traditionnaliste (42\% des sites analysés), regroupe les sites qui pratiquent le e-commerce et qui mettent l'accent sur l'esthétique mais ayant passé le cap de la vente en ligne. Ces sites n'ont pas développé toutes les possibilités interactives d'Internet mais ont néanmoins un degré d'utilisabilité moyen qui permet une navigation agréable. Le site de Lanvin illustre bien les propriétés de cette catégorie.

La deuxième catégorie de cette typologie a été baptisée les E-shops artistiques et regroupe seulement $8 \%$ des sites analysés. Ces sites pratiquent le e-commerce mais sont caractérisés par un niveau d'utilisabilité particulièrement bas. Il s'agit donc de sites, tels que Hermès, qui ont privilégié l'esthétique au détriment de la clarté, sans doute par crainte de s'éloigner trop des valeurs du luxe en proposant la vente en ligne. 
Contrairement aux deux premières, la troisième catégorie de sites (représentant $21 \%$ des sites analysés) regroupe des sites qui ne proposent pas d'e-commerce mais qui mettent l'accent sur les technologies interactives. Différents moyens d'interagir avec la marque sont proposés à travers notamment l'accès à des clubs privilèges ou des conseillers en ligne comme le font Chanel ou Boucheron.

Enfin la dernière catégorie, les Traditionnalistes (représentant 29\% des sites analysés), regroupe des sites qui s'en sont tenus à leur vocation purement communicationnelle. La vente en ligne et les plateformes interactives ne sont pas présentes. On peut cependant apprécier une esthétique recherchée comme par exemple pour la marque Missoni, totalement en ligne avec les supports de communication hors ligne.

\begin{tabular}{|c|c|c|}
\hline \multicolumn{3}{|c|}{ Tableau récapitulatif des résultats } \\
\hline Perspective des managers & Perspectives des consommateurs & Observation des sites Internet \\
\hline $\begin{array}{l}\text { Utilisation essentielle d'Internet pour } \\
\text { les marques de luxe (communication } \\
\text { des valeurs des marques) }\end{array}$ & 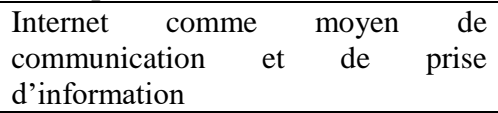 & $\begin{array}{l}4 \text { groupes de sites (selon leur niveau } \\
\text { d'interactivité, d'utilisabilité et la } \\
\text { présence de vente en ligne) }\end{array}$ \\
\hline $\begin{array}{l}\text { Optimisation et maximisation des } \\
\text { nouvelles technologies (vidéos, son, } \\
\text { actualisation) }\end{array}$ & $\begin{array}{l}\text { Internet: support essentiel pour } \\
\text { diffuser l'univers de la marque, son } \\
\text { histoire, ses valeurs }\end{array}$ & $\begin{array}{l}\text { E-shop traditionnalistes ( } 42 \% \text { des sites) : } \\
\text { E-commerce, Interactivité faible et } \\
\text { moyenne, Utilisabilité moyenne } \\
\text { Exemple : Lanvin, Fred, Emilio Pucci }\end{array}$ \\
\hline $\begin{array}{l}\text { Réflexion autour de la mise en relation } \\
\text { du consommateur avec la marque }\end{array}$ & $\begin{array}{lcc}\text { Perceptions } & \text { encore } & \text { mitigées } \\
\text { concernant le } & \text { e-commerce } & \text { dans le } \\
\text { luxe }\end{array}$ & $\begin{array}{l}\text { E-shop artistiques (8\% des sites) : } \\
\text { E-commerce, Interactivité faible, } \\
\text { Utilisabilité faible } \\
\text { Exemple : Hermès, Jean-Paul Gaultier }\end{array}$ \\
\hline $\begin{array}{l}\text { Développement des possibilités de } \\
\text { contacts et de privilèges : clubs privés } \\
\text { mais rejet des forums de discussion }\end{array}$ & $\begin{array}{l}\text { E-commerce pouvant toucher toutes } \\
\text { les catégories de produits (cohérence } \\
\text { perçue) }\end{array}$ & $\begin{array}{l}\text { Interactifs du luxe ( } 21 \% \text { des sites): } \\
\text { Pas de e-commerce, Interactivité forte, } \\
\text { Utilisabilité moyenne } \\
\text { Exemple : Chanel, Boucheron }\end{array}$ \\
\hline $\begin{array}{l}\text { E-commerce en développement mais } \\
\text { réflexion sur la meilleure façon de le } \\
\text { mettre en place (cohérence des valeurs } \\
\text { des marques) }\end{array}$ & $\begin{array}{l}\text { Préférence pour l'expérience vécue } \\
\text { en boutique (besoin de toucher, } \\
\text { manque de confiance) }\end{array}$ & $\begin{array}{l}\text { Traditionnalistes purs ( } 29 \% \text { des sites) : } \\
\text { Pas de e-commerce, Interacticité faible, } \\
\text { Utilisabilité faible et moyenne } \\
\text { Ex : Missoni, Girard Perregaux }\end{array}$ \\
\hline $\begin{array}{lcc}\text { E-commerce utile pour } & \text { le } \\
\text { développement des politiques CRM }\end{array}$ & $\begin{array}{l}\text { Pas de corrélation entre l'âge des } \\
\text { consommateurs et les perceptions } \\
\text { face à Internet et au e-commerce dans } \\
\text { le luxe }\end{array}$ & \\
\hline
\end{tabular}

\section{Discussion des résultats : quelles évolutions ?}

\subsection{Les évolutions des perceptions des managers}

En quelques années, les managers des marques de luxe ont sensiblement changé d'opinion face à l'utilisation d'Internet, l'intégrant automatiquement dans les stratégies de communication. Alors que seul un groupe sur les trois identifiés dans la typologie développée par Nyeck et Roux en 1997 était ouvert à l'intégration d'Internet, il semble qu'aujourd'hui, à la vue des résultats, l'ensemble des managers y est favorable, avec toutefois deux grandes approches : d'une part, les managers proactifs sur Internet, à l'affut de toutes les possibilités offertes pour le média et, d'autre part, les managers classiques qui utilisent Internet de façon plus basique. Autre changement observé : alors que la perception des managers face à Internet dans le luxe variait selon la catégorie de produits et apparaissait comme risqué pour certaines d'entre elles (Nyeck et Roux, 1997 ; Dall'Olmo Riley et Lacroix, 2003), aujourd'hui, les 
managers estiment que l'outil est compatible pour toutes les marques de luxe. Leur absence sur le web pourrait même être très pénalisante.

Ces évolutions amènent les managers à changer leur mode de raisonnement stratégique. Au lieu de se poser la question : "Faut-il développer une présence en ligne pour les marques de luxe ? », ils s'interrogent sur : «comment mettre en place la stratégie Internet des marques de luxe ? ». Il s'agit de questionnements sur le contenu et la forme des sites. Notamment, la perspective interactive a profondément évolué. La dimension relationnelle prend de plus en plus de place dans l'esprit des managers. L'étude de Nyeck et Roux (1997) avait fait ressortir que, pour la majorité des managers, à cette époque, la communication devait se faire uniquement dans un sens, celui de la marque vers les consommateurs. Seuls les managers « ouverts » considéraient une approche bidirectionnelle. L'évolution est donc flagrante à ce niveau puisque que les marques intègrent aujourd'hui de plus en plus d'outils favorisant les échanges. Par rapport à l'étude de Dall'Olmo Riley et Lacroix (2003), l'évolution est moins nette dans la mesure où les managers avaient déjà décelé à l'époque un potentiel important dans l'outil Internet pour développer des relations avec le consommateur à travers des plateformes interactives. Mais malgré cette conscience de l'intérêt que présentait le canal, peu de sites offraient selon l'étude, de vraies interfaces interactives.

Les résultats de cette recherche montrent de forts changements de perceptions des managers face à l'e-commerce dans le luxe. Le scepticisme mis en avant dans les études de Nyeck et Houde (1996) et de Nyeck et Roux (1997) semble s'être transformé en une réalité qui s'impose aux marques. En l'espace de quelques années, la vision des managers a été dépassée par les faits. Lorsque Kapferer et Bastien (2008) énonçaient qu'Internet devait servir à communiquer et non à vendre pour le luxe, les marques commençaient déjà à intégrer l'ecommerce dans leur stratégie et à y voir un développement quasi-obligatoire dans le futur. Cette recherche va davantage dans le sens de Dall'Olmo, Riley et Lacroix (2003) qui voyaient dans l'e-commerce une opportunité et un développement inévitable. Tout comme de Chernatony (2001) l'avait mis en avant, il semble aujourd'hui très important de vendre autrement sur le net, tout en gardant une cohérence de l'identité de la marque. La difficulté est donc de savoir comment intégrer l'e-commerce dans la stratégie de marque. La création de «wishlist » pourrait en être un exemple.

\subsection{L'évolution des perceptions des consommateurs}

Alors qu'il y a quelques années, de nombreux consommateurs restaient encore sceptiques quant à la compatibilité luxe et Internet, aujourd'hui, les consommateurs sont séduits par l'utilisation d'Internet par les marques de luxe. Internet est vu comme un outil permettant d'accéder à un contenu dense de l'univers de la marque, son historique, les caractéristiques des produits ou encore les prix. Les consommateurs jugent comme très satisfaisant leur recherche d'informations montrant une réelle évolution avec les recherches de Dall'Olmo Riley et Lacroix (2003).

Dans cette même perspective, alors qu'à la fin des années 1990, les perceptions face à l'utilisation d'Internet par les marques de luxe dépendaient de l'âge (les plus de 45 ans étant plus sceptiques) (Nyeck et Roux, 1997), aujourd'hui cette variable n'est plus significative. Ce résultat est cohérent puisque, la population vieillissant, les jeunes des années 1990 sont aujourd'hui des quadragénaires ou quinquagénaires experts dans l'achat en ligne.

Les sites sont perçus comme plus attrayants même si parfois les internautes regrettent l'absence de vente en ligne de leur marque préférée. A ce niveau, les résultats de la recherche marquent un réel changement dans les mentalités. Les consommateurs sont de plus en plus 
demandeurs pour que leurs marques vendent en ligne leurs produits. De plus, les catégories de produits recherchées pour l'e-commerce dans le luxe ne concernent plus uniquement les cosmétiques et les accessoires comme cela était le cas dans les études de Nyeck et Roux (1997), de Dall'Olmo Riley et Lacroix (2003) et de Seringhaus (2005). Aujourd'hui, les consommateurs souhaitent acheter en ligne principalement des vêtements et des accessoires mais considèrent de plus en plus les bijoux. Cependant, même si l'e-commerce dans le secteur du luxe est de plus en plus accepté, les consommateurs gardent toujours une préférence pour l'achat en magasin. Ils ont besoin de sentir et toucher les produits. Ces résultats restent dans la lignée des recherches antérieures et mettent en avant l'importance de l'expérience d'achat (Okonkwo, 2007). Les consommateurs ne choisissent pas l'e-commerce en substitut d'un mode de consommation plus classique mais de façon complémentaire.

\subsection{L'évolution des sites des marques de luxe}

Dall'Olmo Riley et Lacroix (2003) notaient dans leur audit que seuls $70 \%$ des sites de l'échantillon de 54 sites étaient opérationnels (c'est-à-dire présentaient effectivement un contenu). Par exemple, à l'époque de leur étude, Hermès ne possédait pas de site opérationnel ; il s'agissait juste d'une page avec un nom et des visuels. Aujourd'hui tous les sites des marques de luxe sont actifs. D'un point de vue purement visuel, les sites de marque de luxe étaient déjà portés sur l'esthétique puisque les auteurs avaient recensé la présence de photos et d'illustrations dans $72 \%$ des cas. De notre analyse, $100 \%$ des sites audités sont illustrés. Dans l'étude de Dall'Olmo Riley et Lacroix (2003), 25\% des sites analysés disposaient d'un moteur de recherche, notre étude montre que moins de $10 \%$ des sites étudiés n'en disposent pas. Ce type de site fait d'ailleurs l'objet d'une catégorie à part entière appelée E-shops Artistiques. De Plus, alors qu'à l'époque, seuls $15 \%$ des sites proposaient la vente en ligne, ce pourcentage est passé à 50\% selon notre analyse, marquant une réelle évolution. Du point de vue de l'interactivité, il y avait déjà à l'époque des légères tendances à la communication bidirectionnelle avec des possibilités de contact avec la marque $(25 \%$ des sites audités par Dall'Olmo Riley et Lacroix, 2003) mais ces possibilités se sont largement accrues avec le temps puisque cet onglet «contact» est présent désormais dans plus de $90 \%$ des cas. On peut également observer l'existence de clubs «privilège » avec des pages réservées uniquement aux clients des marques. Enfin on constatait à l'époque de nombreuses difficultés de chargement dues à des pages trop lourdes, ce problème ne semble plus se manifester au travers des dernières analyses.

De nettes évolutions sont ainsi constatées avec une propension vers la vente en ligne de plus en plus marquée et une tendance accrue pour l'interactivité. Ces évolutions des sites Internet confirment l'opinion des managers qui ne se demandent plus s'ils doivent être sur Internet mais bien comment y affirmer leur présence.

\section{Limites et futures voies de recherche}

Bien que de nombreuses précautions aient été prises, cette recherche présente plusieurs limites. La première concerne l'échantillon utilisé pour l'analyse quantitative des perceptions des consommateurs de luxe. Pour cette étude, des clients de l'enseigne multi-marques F. Ferent ont été interrogés. Il serait intéressant dans une prochaine étude d'étudier les perceptions des clients d'une marque de luxe en particulier. La seconde limite porte sur le caractère ponctuel de l'étude. En effet, ayant montré dans cette recherche qu'en quelques années seulement, les avis des consommateurs et des managers avaient fortement et donc 
rapidement évolué, l'étude devrait être reconduite tous les 3 à 5 ans maximum et pourrait avoir un caractère longitudinal.

Cette perspective d'évolution rapide nous amène à réfléchir sur d'autres pistes de recherche afin de mieux appréhender cet environnement changeant et d'y répondre par des stratégies de marque adéquates. En effet, cette ouverture vers la vente en ligne doit-elle se faire sur l'ensemble des produits de la marque ou sur une sélection particulière ? Le cas échéant, quels sont les critères de sélection de ces produits propices à la vente en ligne ? Vaut-il mieux pratiquer la vente en ligne sur le site de la marque ou sous-traiter à des enseignes multimarques en ligne? De nombreuses questions sont donc soulevées à l'issue de cette réflexion et confirme donc le potentiel de recherches de cette problématique.

\section{Bibliographie}

ALLERES D. (1991), «Spécificités et Stratégies Marketing des différents univers du luxe », Revue Française du Marketing, Vol. 132-133, No. 2-3, p. 71-95.

ALLERES D. (1997), Luxe ... Stratégies-Marketing, Paris, Economica.

ATWAL G. et WILLIAMS A. (2009), «Luxury brand marketing - the experience is everything! », Brand Management, Vol. 16, p. 338-346.

BECHTOLD M. (1991), «Le paradoxe du luxe», Revue Française du Marketing, Vol. 132133, No. 2-3, p. 41-43.

CASTAREDE J. (2004), Le luxe, Que sais-je ?, Paris, Presses Universitaires de France

CHADHA R. et HUSBAND P. (2006), The Cult of Luxury Brands: Inside Asia's Love Affair with Luxury, Nicholas Brealey International, London, Boston

CHAFFEY D., MAYER R., JOHNSTON K. and ELLIS-CHADWICK F. (2003) Internet marketing, Essex: Prentice Hall, Financial Times/ Prentice Hall, Harlow.

CHEVALIER M. et MAZZALOVO G. (2008), Management et marketing du luxe, Dunod.

DALL'OLMO RILEY F. et LACROIX C. (2003), "Luxury branding on the Internet: lost opportunity or impossibility? », Marketing Intelligence \& Planning, Vol.21, No.2, p. 96-104.

DE CHERNATONY L. (2001), "Succeeding with Brands on the Internet », Brand Management, 8 (February), Vol. 3, p. 186-195.

DUBOIS B. et LAURENT G. (1993), «Is there a Euro consumer for luxury goods? », European Advances in Consumer Research, Vol. 1, p. 58-69.

HEILBRUNN B.(2002), Le luxe est mort, vive le luxe! Le marché du luxe à l'aune de la démocratisation dans, Le Luxe, essais sur la fabrication de l'ostentation, Edition du Regard (IFM), p. 353-369.

JOLIBERT A. et JOURDAN P. (2011), Marketing Research, Paris, Dunod JOLLIFE, I.T. (2002), Principal Componant Analysis, Springer, New-York. KAPFERER J.-N. et BASTIEN V. (2008), Luxe oblige, Eyrolles Editions d'Organisation.

KELLER K. L. (2009), « Building strong brands in a modern marketing communications environment », Journal of Marketing Communications, Vol. 15, No. 2-3, p. 139-155.

LARBANET C. et LIGIER B. (2010), The Internet use by the Luxury industry An interactive tool for a very demanding sector, Saarbrucken, Lambert Academic Publishing.

LEMOINE J-F. (2012), « À la recherche d'une meilleure compréhension du comportement des internautes », Management \& Avenir, Vol. 8, No. 58, p. 116-119.

LIPOVETSKY G. et ROUX E. (2003), Le luxe éternel: de l'âge du sacré au temps des marques, Gallimard. 
MEYER N., MAUMY-BERTRAND M. et BERTRAND F. (2010), Comparaison de variantes de régressions logistiques PLS et de régression PLS sur variables qualitatives : application aux données d'allélotypage, Journal de la Société Française de Statistique, 151, 2.

NUEMO J.L. et QUELCH J.A. (1998), « The mass marketing of luxury », Business Horizons, Vol. 41, No. 6, p. 61-8.

NYECK S. (2004), « Luxury Brands Online and Offline: The Case of French Brands », The European Retail Digest, Janvier, 41.

NYECK S. et HOUDE S. (1996), «L'implantation des entreprises de luxe sur le www: un état des lieux », Décisions Marketing, Vol. 9, Septembre-Décembre, p. 51-60.

NYECK S. et ROUX E. (1997), WWW as a communication tool for luxury brands: compared perceptions of consumers and managers, 24th International Research Seminar in Marketing, Communications and Consumer Behavior, june 3-6, La Londe les Maures, IAE Aix-FNEGE, 296-316.

OKONKWO U. (2007), Luxury fashion branding, trends, tactics, techniques, Palgrave McMillan.

OKONKWO U. (2009), «Sustaining Luxury Brands on the Internet », Journal of Brand Management, Vol. 16, No. 5/6, p. 302-310.

OKONKWO U. (2010), Luxury Online, Style, Systems, Strategies, Palgrave McMillian.

PHAU I. et PRENDERGAST G. (2000), « Consuming luxury brands: The relevance of the `Rarity Principle », The Journal of Brand Management, Vol. 8, No. 2, p. 122-138.

ROUX E. et FLOCH J.-M. (1996), «Gérer l'ingérable : la contradiction interne de toute maison de luxe », Décisions Marketing, Vol. 9, p. 15-25.

SERINGHAUS F.H.R. (2005), «Selling Luxury Brands Online », Journal of Internet Commerce, Vol. 4, No. 1, p. 1-25.

VEG-SALA N. et GEERTS A. (2012), «Gestion de la cohérence des récits des marques de luxe sur Internet : étude sémiotique et analyse comparée des secteurs de la maroquinerie et de la joaillerie », Revue Française du Marketing, Vol. 233, No. 3/5, p. 5-26. 